Artículo Original

\title{
Dolor fantasma en niños y jóvenes amputados congénitos: prevalencia $y$ características clínicas
}

ELIZABETH FLORES $\mathrm{S}^{1}$, DANIELA GARCÍA P²

${ }^{1}$ Residente Programa Medicina Física y Rehabilitación. Universidad del Desarrollo. ${ }^{2}$ Instituto Teletón Santiago.

Recibido: 23-01-2019 Aprobado: 17-05-2019

Correspondencia:

Dra. Elizabeth Flores S eliflores@teleton.cl

\section{ABSTRACT \\ Phantom pain in children and youth with congenital amputations: prevalence and clinical characteristics}

Introduction: Pain is a crippling pathology, which has been addressed in children only in recent times, and phantom pain in congenital amputees was a complication unthought to exist some years ago, considering theories that described that they lacked biological maturity enough to experience it. Currently, there is literature that recognizes a prevalence of phantom pain of 3.7 to $20 \%$ in congenital amputees. Overall objective: To evaluate the in vivo amputee population of Instituto Teletón Santiago, to determine incidence, lapses prevalence and clinical characteristics of phantom pain in these patients. Patients and Methods: A prevalence descriptive study was carried out, with a review of the clinical files to register demographic and clinical variables and application of a telephone survey to the total number of Instituto Teletón Santiago patients who agreed to participate and had the consent of their parents. We included all patients aged 10 years and over; excluding those with associated hearing loss, or inability to contact for administrative reasons. Results: 57 patients, mean age 18,6 years, $59,6 \%$ with longitudinal deficiency. No patient refered phantom pain during de last month in the study population. The lapsic prevalence was $7,0 \% .24,6 \%$ of the population studied reported phantom feeling at som time in their lives and 5,3\% had phantom pain during the last year. Conclusion: There is phantom pain in the population of congenital amputees in the prevalence ranges found in the international literature contributing to the existing evidence. This is the first study in our country that evaluates pain in congenital deficiencies and constitutes a baseline for additional studies.

Key words: Phantom pain, prevalence, congenital amputees, limb loss.

\section{RESUMEN}

Introducción: El dolor es una patología discapacitante, que en niños está siendo abordada en los últimos tiempos, siendo el dolor fantasma en amputados congénitos una complicación impensada hace algunos años, considerando teorías que describían que carecían de la madurez biológica suficiente para 
experimentarlo. Actualmente, existe literatura que reconoce una prevalencia de dolor fantasma de 3,7 a $20 \%$ en la población de amputados congénitos. Objetivo general: Evaluar población de amputados congénitos del Instituto Teletón Santiago para determinar incidencia, prevalencia lápsica y características clínicas del dolor fantasma. Pacientes y Métodos: Estudio descriptivo de prevalencia, con revisión de ficha clínica para registro de variables demográficas y clínicas, y aplicación de encuesta telefónica al total de pacientes que asintieran y contaran con el consentimiento de sus padres. Se incluyó a todos los pacientes de 10 o más años; se excluyó a los con hipoacusia asociada o con imposibilidad administrativa para contactarlos. Resultados: 57 pacientes, edad promedio 18,6 años, 59,6\% con deficiencia longitudinal. Ningún paciente presentó dolor fantasma en el último mes en la población estudiada. La prevalecia lápsica fue de 7,0\%. Un 24,6\% refirió además presentar sensación fantasma alguna vez en su vida y un 5,3\% haber presentado dolor en el último año. Conclusión: Existe dolor fantasma en la población de pacientes amputados congénitos en los rangos de prevalencia encontrados en la literatura internacional contribuyendo a la evidencia existente. Este es el primer estudio en nuestro país que evalúa dolor en deficiencias congénitas y constituye una línea de base para estudios adicionales.

Palabras clave: Amputados congénitos, deficiencias, prevalencia, dolor fantasma.

\section{Introducción}

La causa más frecuente de amputaciones de las extremidades en la infancia es la de origen congénito ${ }^{1}$. Las deficiencias congénitas (DC) pueden atribuirse a diferentes causas: medicamentos, lesiones vasculares como síndrome de banda amniótica, malformaciones vasculares, factores genéticos y teratógenos prenatales $^{2}$, aunque, en la mayoría la causa es todavía desconocida ${ }^{2,3}$

Las DC de extremidades son catalogadas según la clasificación de la Sociedad Internacional de Órtesis y Prótesis (ISPO) transversales, donde la extremidad aparece amputada en sentido transversal a su eje, o longitudinales donde la ausencia de segmentos de la extremidad es en el eje longitudinal ${ }^{4,5}$.

En el caso de las DC de extremidades, se describe la presentación unilateral como la más frecuente ${ }^{5}$. Las extremidades superiores son las más afectadas, siendo el tipo más común la DC transversal de antebrazo izquierdo ${ }^{5-8}$.

En Estados Unidos, la prevalencia de pacientes amputados en 2005 era de 1,6 millones y se estima que serán 3,6 millones en 2050, dado el aumento de amputados disvasculares ${ }^{9}$. En el Instituto Teletón Santiago (ITS) se atienden actualmente 453 pacientes con diagnóstico de DC, lo que corresponde al 1,67\% del total de pacientes atendidos en los Institutos Teletón a nivel nacional ${ }^{10}$.

Los problemas inherentes a las DC son, entre otros, los desbalances motores y funcionales y, recientemente descrito, la existencia de sensación y dolor fantasma.

La primera vez que se describió médicamente la sensación de tener una extremidad posterior a la amputación de ésta en adultos fue en el siglo XVI, por el cirujano francés Ambroisie Paré (1510-1590) ${ }^{11,12}$. El término de dolor fantasma fue acuñado por Mitchell en 1866, quien describió los síntomas fantasmas como réplicas fantasmales de la extremidad perdida, algunas de ellas dolorosas y otras no ${ }^{13,14}$.

En cuanto al dolor en niños, han existido múltiples teorías; una de ellas describía que percibían el dolor con menor intensidad, debido a la inmadurez biológica de su sistema nervioso central, suponiendo que tenían baja 
capacidad en recordar experiencias dolorosas, así como un umbral de dolor más alto con una tolerancia mejor que en el adulto ${ }^{15,16}$. Actualmente, las teorías han cambiado y se reconoce que el dolor constituye un problema común que pueden sufrir niños y adolescentes ${ }^{15,17,18}$

A pesar del gran número de investigaciones sobre la sensación y dolor fantasma en adultos amputados, existe escasa información sobre niños y adolescentes amputados. Una explicación, podría ser que los niños no tienen la habilidad verbal para describir la sensación o dolor fantasma ${ }^{19}$.

Analizando los artículos publicados, destaca que la sensación fantasma es más común en niños con amputaciones traumáticas, existiendo estudios en la que aparece en el 100\% de estos, mientras que $83-100 \%$ de ellos experimentan dolor fantasma ${ }^{20,21}$. A nivel nacional, el año 2013, se realizó un estudio en niños y adolescentes amputados adquiridos donde la incidencia de dolor fantasma fue de $11 \%$ y la prevalencia de $63 \%{ }^{8}$. Uno de los artículos que incluyó mayor número de pacientes fue el de Melzack y cols., que estudiaron a 125 personas con amputación de extremidades a través de un cuestionario, siendo la edad promedio 14,7 años. La sensación fantasma fue experimentada por $20 \%$ de los sujetos con DC y en $50 \%$ de los que tuvieron amputación antes de los 6 años, describiéndose como dolorosa en el $20 \%$ y $42 \%$ de los grupos, respectivamente. Así mismo, se observó que en la mayoría de los casos, la sensación fantasma persistía en la adolescencia y adultez ${ }^{22}$.

Como se puede desprender de los artículos antes mencionados, actualmente se cuenta con evidencia científica que corrobora la presencia de dolor fantasma en DC. Sin embargo, la sospecha clínica es baja. Esto puede llevar a un subdiagnóstico y falta de manejo del problema en estos pacientes. El presente estudio tiene como objetivo principal determinar la incidencia, prevalencia y características clínicas del dolor fantasma en amputados congénitos y caracterizar esta población desde el punto de vista clínico y demográfico, con la finalidad de dar a conocer y mejorar la comprensión de esta complicación en nuestro país y dar pie a futuras investigaciones.

\section{Materiales y Métodos}

\section{Diseño del estudio}

Estudio descriptivo, de prevalencia, retrospectivo.

\section{Población estudiada}

Niños y jóvenes con diagnóstico de DC que recibieron atención en ITS y que en el año 2016 tuvieron 10 años y más. Se excluyeron pacientes con deterioro cognitivo y/o déficit auditivo asociado que les impedía contestar la encuesta telefónica y aquellos que por causas administrativas no se pudieron contactar, eligiendo edad mayor a 10 años para mejorar comprensión y disminuir sesgo de memoria.

\section{Instrumentos de medición}

Se seleccionó una encuesta para detectar la presencia de dolor fantasma que fue utilizada y validada previamente en un estudio en amputados adquiridos realizado en Instituto Teletón Santiago; este instrumento se basó en escalas de dolor neuropático y fantasma admitidas para la población adulta ${ }^{8}$, ya que no se cuenta con escalas de dolor neuropático pediátricas. Se utilizó el mismo instrumento para mantener la metodología y ser una continuidad en la investigación en niños con amputación, adaptándose la pregunta de amputación adquirida a congénita y acotando el período evaluado del último año al último mes para disminuir el sesgo de memoria.

\section{Variables estudiadas}

Revisión de base de datos Cubo Olap para obtener número telefónico y de ficha clínica de la población total. A los pacientes con DC y consentimiento y asentimiento, se les aplicó encuesta telefónica modificada ${ }^{8}$.

Se realizó revisión de fichas clínicas de los pacientes que respondieron a la encuesta y cumplían criterios de inclusión. Se obtuvieron las siguientes variables demográficas: fecha de nacimiento, sexo, previsión y residencia. También se registraron los siguientes antecedentes clínicos: edad de inicio de uso de prótesis, número y nivel de amputación, extremidad afectada, y fecha de una posible amputación quirúrgica posterior (cirugía de reconversión). 
La encuesta se realizó entre marzo de 2016 y mayo de 2017 y evaluó la presencia de sensación fantasma, dolor fantasma, primer y último episodio de dolor, intensidad del dolor, características y gatillantes. Además, se preguntó por las horas diarias de uso de prótesis, cuando correspondía.

\section{Análisis de resultados}

Las variables fueron tabuladas en planilla Excel y se procesaron con software STATA 14.

Las tasas se calcularon de la siguiente forma:

$$
\text { Prevalencia lápsica }=\frac{\begin{array}{c}
N^{\circ} \text { pacientes que han tenido } \\
\text { dolor alguna vez en su vida }
\end{array}}{\begin{array}{c}
N^{\circ} \text { total de pacientes } \\
\text { encuestados }
\end{array}} * 100
$$

\section{Consideraciones éticas}

Se cauteló la confidencialidad de la información y participación voluntaria, mediante asentimiento y consentimiento informado oral, a los menores de edad y padres antes de realizar la encuesta telefónica, con grabación de los datos registrados.

Los pacientes que reportaron dolor al momento de la encuesta fueron derivados a control médico en ITS. El estudio contó con certificado de autorización del Comité Ético Científico de Teletón Chile (71/2016), sin identificarse conflictos de interés.

\section{Resultados}

Se llamó al total de pacientes registrados en la base de datos Cubo OLAP. Sólo 77 $(34,1 \%)$ del total de pacientes pudieron ser contactados telefónicamente. El detalle de los llamados se presenta en la Figura 1. Se revisaron 57 fichas clínicas de los pacientes que contestaron la encuesta. Cabe destacar que 13 de los pacientes que respondieron la encuesta, estando diagnosticados de deficiencia

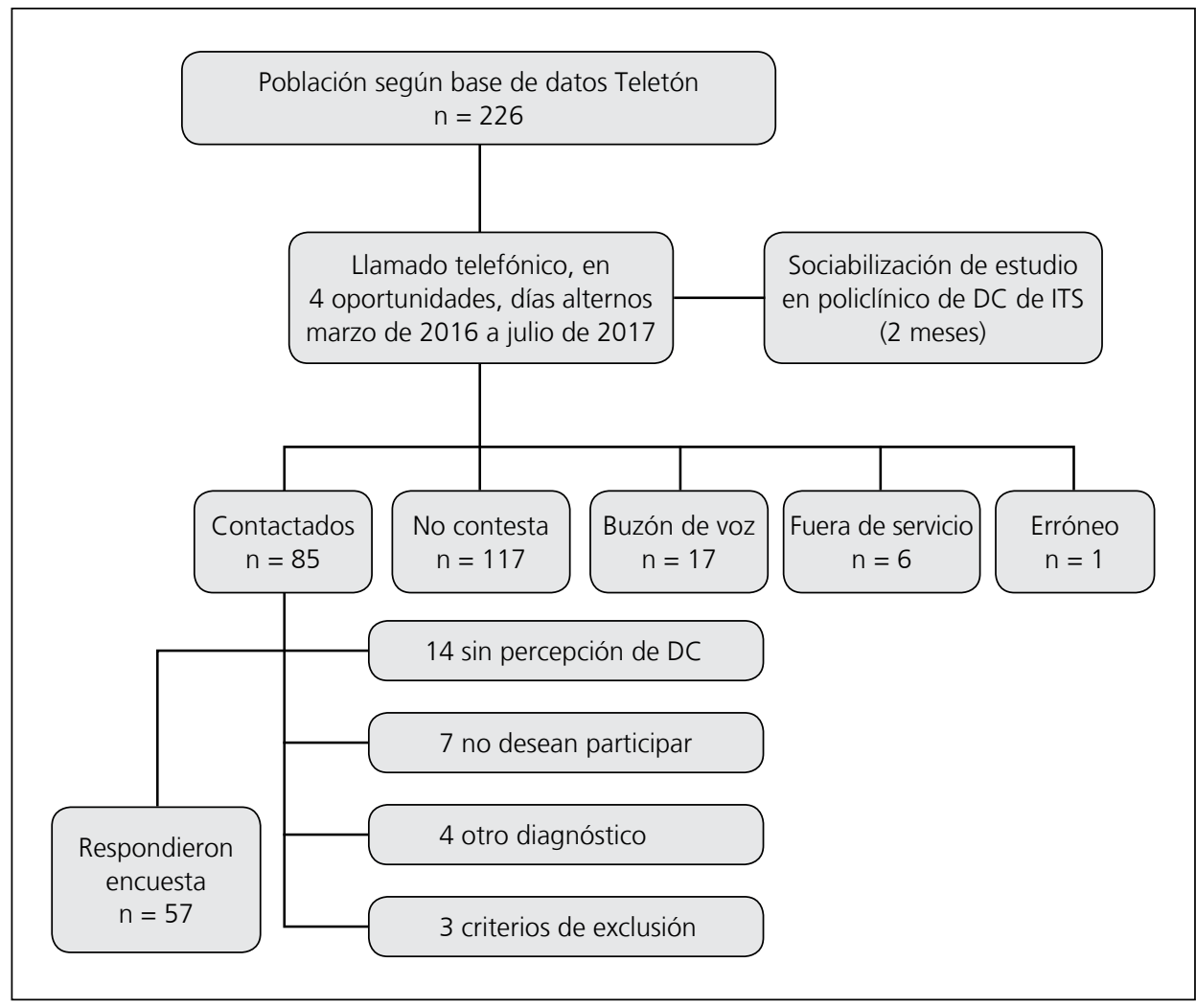

Figura 1. Identificación de la población encuestada. 
congénita, al momento de realizar la pregunta de percepción de la deficiencia respondieron que no tenían deficiencia alguna, presentándose esto en usuarios con deficiencia longitudinal no evidenciable como ausencia de una extremidad al examen físico, por lo que se los excluyó del estudio.

\section{Características demográficas y clínicas registradas}

El detalle de los 57 pacientes encuestados se presenta en la Tabla 1. La amputación más frecuente fue de tipo longitudinal, seguido por el transversal. Las extremidades superiores fueron afectadas en un $68,5 \%$, siendo más frecuente la amputación transversal de antebrazo izquierdo. Los detalles de tipo y localización de DC se especifican en la Tabla 2. Un $71,9 \%$ de los pacientes presenta compromiso de una extremidad, y un $28,1 \%$ de dos o más extremidades.

En cuanto al uso de prótesis, un 33,0\% de los encuestados reportó utilizarla, con una mediana de 10 meses de edad para la entrega de la primera prótesis. En DC de extremidades superiores un $25,4 \%$ reportó el uso de prótesis, correspondiendo de estos a DC transversal de antebrazo en un $92,0 \%$. Con respecto a las extremidades inferiores un $27,7 \%$ de los encuestados manifestó uso de prótesis, siendo todos del tipo de DC longitudinal y posterior a cirugía de reconversión.

\section{Sensación y dolor fantasma (encuesta telefónica)}

Del total de pacientes encuestados, un 24,6\% (14 pacientes) había tenido sensación fantasma y un $7,0 \%$ (4 pacientes) refirió sufrir dolor fantasma alguna vez en su vida, constituyendo la prevalencia lápsica. Ningún paciente presentó dolor fantasma en el último mes, al momento de la encuesta. Un 5,3\% presentó dolor el último año. De los 4 pacientes que reportaron haber sufrido dolor fantasma, todos presentaban DC de extremidades superiores, tres DC de tipo longitudinal en una extremidad y uno DC de tipo transversal de mano, además de un pie. Ninguno requirió cirugía de reconversión y no reportaron uso
Tabla 1. Descripción demográfica con indicación de uso de prótesis

\begin{tabular}{|lcc|}
\hline Característica & $\mathbf{n}$ & \% * \\
\hline $\begin{array}{l}\text { Edad (años) } \\
\quad \text { Promedio (rango) }\end{array}$ & 18,6 & $(12-25)$ \\
$\begin{array}{l}\text { Sexo } \\
\text { Hombres }\end{array}$ & 25 & 43,9 \\
\hline $\begin{array}{l}\text { Previsión } \\
\text { Fonasa }\end{array}$ & 53 & 93,0 \\
$\begin{array}{l}\text { Ciudad de residencia } \\
\quad \text { Santiago }\end{array}$ & 45 & 78,9 \\
\hline
\end{tabular}

*Porcentaje calculado sobre 57 pacientes.

Tabla 2. Tipo y localización de deficiencias congénitas

\begin{tabular}{|lrr|}
\hline & $\mathbf{n}$ & $\%$ \\
\hline Tipo & & \\
$\quad$ Amputación transversal & 22 & 38,6 \\
Amputación longitudinal & 34 & 59,6 \\
Ambas & 1 & 1,8 \\
Total & 57 & 100,0 \\
\hline Localización & & \\
Derecha superior & 12 & 21,1 \\
Izquierda superior & 23 & 40,4 \\
Superiores & 4 & 7,0 \\
Derecha inferior & 2 & 3,5 \\
Izquierda inferior & 3 & 5,3 \\
Derecha superior e inferior & 1 & 1,8 \\
Derecha superior e izquierda inferior & 2 & 3,5 \\
Cuatro extremidades & 8 & 14,0 \\
Tres extremidades & 2 & 3,5 \\
\hline
\end{tabular}

de prótesis. Dos de ellos manifestaron también sentir sensación fantasma.

\section{Discusión}

En el presente estudio revisamos la prevalencia de sensación y dolor fantasma de los niños con DC de extremidades, que se controlan o controlaban en ITS. También se realizó una descripción demográfica y clínica de la población en cuestión.

Las características clínicas encontradas en los pacientes encuestados son similares a las descritas en la literatura. Por ejemplo, en un estudio realizado en un centro de rehabilitación 
en India, que analizó las variables demográficas y clínicas de 200 pacientes con DC, se encontró como tipo de deficiencia más frecuente la transversal de antebrazo ${ }^{23}$, al igual que en este estudio. Este mismo resultado se presenta en otras publicaciones con menor cantidad de pacientes $^{5,24}$. El compromiso de las extremidades superiores fue más frecuente que el de las inferiores, al igual que en la literatura ${ }^{23-26}$. De este análisis podemos desprender que a pesar de que el número de pacientes encuestados no contiene la población total, sus características son consistentes con los reportados a nivel internacional.

En cuanto al uso de prótesis, la edad promedio recomendada para iniciar su uso varía de los 6 a los 12 meses $^{27,28}$, dependiendo del tipo y nivel de DC. En nuestros encuestados, la primera prótesis fue entregada en promedio a los 10 meses, cumpliendo con los estándares internacionales actuales; probablemente se debe a que el Instituto Teletón cuenta con un equipo clínico multidisciplinario especializado en el abordaje de niños con amputaciones. Sólo un tercio de los pacientes del estudio utilizaban prótesis. Esta baja adherencia es concordante con la literatura, en nuestro caso debido a que la población correspondió principalmente a DC de extremidades superiores e incluía DC longitudinales, donde la evidencia muestra que las actividades de la vida diaria se desarrollan sin problema a pesar de la deficiencia ${ }^{1,27}$.

Con respecto a la sensación fantasma, distintos estudios han reportado que está presente desde 7,4 a 19,7\% de los pacientes con DC. El dolor fantasma está reportado entre un 3,7 y un $20,0 \%$ de estos pacientes ${ }^{14,17}$. El mayor estudio publicado fue realizado por Ronald Melzack y colaboradores en 1997 e incluyó a 125 pacientes, y 66 de ellos tenían como diagnóstico una DC. En nuestro estudio, la prevalencia de dolor fantasma se encuentra dentro del rango publicado en la literatura, pero el porcentaje de sensación fantasma es incluso mayor al descrito. Creemos incluso que estos resultados, podrían ser aún mayores ${ }^{29}$. Esto último porque, en nuestro caso, no pudimos contactar por dificultades en la localización telefónica a la totalidad de pacientes con DC.
Otra razón podría ser que la sensación y dolor fantasma se presentan de forma esporádica, y suelen ser de corta duración ${ }^{14,22}$, por lo que el sesgo de la memoria podría influir al momento de realizar la encuesta.

Dado el pequeño número de pacientes que reportaron dolor fantasma al momento de la encuesta, no es posible obtener conclusiones significativas de esta población. Sin embargo, se evidencian ciertas tendencias concordantes con la literatura. En los casos publicados el dolor fantasma aparece prioritariamente en DC de extremidades superiores de presentación unilateral, en que predominantemente no existe uso de prótesis ${ }^{22,30,31}$, características compartidas por nuestro estudio. En cuanto a la presentación del dolor, la literatura menciona una aparición de menos de una vez al mes, lo que pudiera estar relacionado con la ausencia de incidencia de dolor que encontramos.

Tanto nuestros resultados, como los publicados a nivel internacional apoyan la existencia de dolor y sensación fantasma. Los reportes a lo largo de la literatura han llevado a una serie de expertos a inferir que las representaciones corporales corticales están suscritas a un modelo genéticamente codificado ${ }^{17,32-34}$. Nos parece interesante presentar la hipótesis publicada en una revisión del año 2006, que recopila las experiencias y publicaciones en deficiencias congénitas presentadas en la literatura a lo largo de los años ${ }^{31}$. Este trabajo concluye que existirían dos etapas en el desarrollo de la imagen corporal. La primera, donde los aspectos relacionados con ésta se aprenden bilateralmente en el útero, como resultado de la actividad muscular voluntaria, prioritariamente unilateral en un inicio, y la información sensorial ${ }^{31}$, permitiendo a nuestro juicio explicar la sensación fantasma cuando existe la extremidad contralateral, con presentación de la sintomatología en la infancia temprana. La segunda etapa, se desarrollaría durante la primera década de vida, estando determinada por las aferencias sensitivas y la retroalimentación propioceptiva del cuerpo, lo que explicaría la emergencia de la sensación fantasma durante la niñez y en algunos casos la adolescencia, consolidándose 
durante las primeras décadas a causa de las sensaciones somestésicas y kinestésicas de las extremidades existentes, la información visual y las neuronas en espejo ${ }^{31,35}$. Esto podría dar respuesta a los casos de sensación fantasma en donde no existe miembro contra lateral presente.

En etapas posteriores de la vida se ha visto también que la sensación fantasma puede ser inducida por el uso de prótesis ${ }^{31}$. Varios estudios muestran además que, bajo ciertas condiciones, es posible incorporar objetos inanimados a la percepción de la imagen corporal $^{23,34,36-38}$. Por las características de esta investigación, no fue posible evaluar esta hipótesis, pero concordamos con lo presentado por el autor, principalmente con las causas posnatales donde hemos comprobado a través de la experiencia clínica, que el uso precoz de la prótesis se incorpora al esquema e imagen corporal del niño.

Dentro de las principales limitaciones de este estudio, destaca la cantidad de pacientes que no fue posible localizar telefónicamente. Esto deja de manifiesto un importante déficit en la forma en que actualmente se pesquisan y registran los datos de contacto. Otra limitación fue el pequeño número de pacientes con dolor, lo que no permite sacar mayores conclusiones de este grupo. Por último, mencionar el carácter retrospectivo del estudio y los sesgos para registrar la presencia de dolor que se mencionaron anteriormente.

\section{Conclusión}

Dadas las pocas publicaciones que existen actualmente con respecto al dolor fantasma en niños con DC, el presente estudio es un importante aporte y constituye el primer estudio realizado en nuestro país. Creemos que contribuirá a concientizar al equipo médico y clínico en la existencia de esta complicación, para así pesquisarla y manejarla precozmente. Nuestra sugerencia es la búsqueda activa en cada control fisiátrico, a través de preguntas directas al paciente y su familia. A su vez, los resultados obtenidos pueden servir de base para futuros estudios.

\section{Referencias bibliográficas}

1. Vasluian E, van der Sluis C, van Essen A, Bergman, Dijkstra1, Reinders-Messelink A, et al. Birth prevalence for congenital limb defects in the northern Netherlands: a 30-year population-based study. BMC Musculoskeletal Disord 2013; 14: 323.

2. Le J, Scott-Wyard R. Pediatric Limb Differences and Amputations. Phys Med Rehabil Clin N Am 2015; 26 : 95-108

3. Yoon P, Rasmussen S, Lynberg M, Moore C, Anderka $\mathrm{M}$, Carmichael S, et al. The national birth defects prevention study. Public Health Rep 2001; 116: 32-40.

4. Varma P, Stineman M, Dillingham. T. Epidemiology of limb loss. Phys Med Rehabil Clin N Am 2014; 25: 1-8.

5. Fattah Al-Worikat A, Dameh W. Children with limb deficiencies: Demographic characteristics. Prosthet Orthot Int 2008; 32: 23-8.

6. Boonstra A, Rijnders L, Groothoff J, Eisma W. Children with congenital deficiencies or acquired amputations of the lower limb: Functional aspects. Prosthet Orthot Int 2000; 24: 19-27.

7. Bryant P, Pandian G. Acquired limb deficiencies. Acquired limb deficiencies in children and young adults. Arch Phys Med Rehabil 2001; 82 (Suppl 1): 53-8.

8. Velasco A, García D, Solís F, San Martín P. Dolor fantasma en niños y jóvenes amputados adquiridos: prevalencia y características clínicas. Rehabil. Integral 2015; 10: 8-16.

9. Ziegler-Graham K, MacKenzie EJ, Ephraim PL, Travison TG, Brookmeyer R. Estimating the prevalence of limb loss in the United States: 2005 to 2050. Arch Phys Med Rehabil 2008; 89: 422-9.

10. Cubo olap. Sistema informático Teletón Chile.

11. Weeks S, Anderson-Barnes V, Tsao J. Phantom limb pain Theories and Therapies. Neurologist 2010; 16: 277-86.

12. Nikolajsen L, Jensen T. Phantom limb pain. Br J Anaesth 2001; 87: 107-16.

13. Mitchel S. The case of George Dedlow, The Atlantic Monthly. 1866; 18: 1-11.

14. Wilkins K, Krista L, McGrath P, Finley G, Katz J. Phantom limb sensations and phantom limb pain in child and adolescent amputees. Pain 1998; 78: 7-12.

15. Tesler M, Ward J, Saavedra M, Gibbons P. Developing an instrument for eliciting Children's description of pain. Perceptual and motor skills 1983; 56: 315-21.

16. Sánchez F, Pellegrini F, Malmierca A. Valoración del dolor en pediatría. Curso de formación continuada en pediatría. Pediatría integral 2008; 3-17. 
17. Roth-Isigkeit. Almost one third of German children and adolescents suffer from chronic pain. Póster presentado en el 4th Congress of the European Federation of IASP Chapters. Praga. Septiembre, 2003.

18. Groholt E, Stigum H, Nordhagen R, Kohler L. Recurrent pain in children, socio-economic factors and accumulation in families. Eur J Epidemiol 2003; 18: 965-75.

19. Easson W, Sask S. Body image and self-image in children. Arch Gen Psychiatry. 1961; 4: 619-621.

20. Ritchie JA. Nursing the child undergoing limb amputation. Am. J. Matem. Child Nurs 1980; 5: 114-120.

21. Krane EJ, Heller LB. The prevalence of phantom sensations and phantom pain in pediatric amputees. J Pain Symptom Manage 1995; 10: 21-9.

22. Melzack R, Isreal R, Lacroix R, Shultz G. Phantom limbs in people with congenital limb deficiency or amputation in early childhood, Brain 1997; 120: 1603-20.

23. Jain SK. A study of 200 cases of congenital limb deficiencies. Prosthet Orthot Int 1994; 18: 174-9.

24. Kuyper MA, Breedijk M, Mulders AHM, et al. Management of children in the Netherlands with upper limb deficiencies. Prosthet Orthot Int 2001; 25: 228-34.

25. Bochman D. Prostheses for the limb-deficient child. In Kostuik JP (Ed): Amputation surgery and rehabilitation. Churchill Livingstone 1981. Pg. 293-310.

26. Scotland TR, Galway HR. A long-term review of children with congenital and acquired upper-limb deficiency. J Bone Joint Surg 1983; 65B:346-9.

27. Jain S. Rehabilitation in limb deficiency. 2. The paediatric amputee. Arch Phys Med Rehabil 1996; 77 (3 Suppl): S9-S13.

28. Hirons RR, Williams KB, Amor RF, Day HJB. The prosthetic treatment of lower limb deficiency. Prosthet Orthot Int 1991; 15: 112-6.

29. Simel M. The conditions of occurrence of phantom limbs. Proceedings of the American philosophical society. JSTOR 1958; 102: 492-500.

30. Weinstein S, Sersen EA, Vetter RJ. Phantoms and somatic sensations in cases of congenital aplasia. Cortex 1964; 1: 276-90.

31. Price EH. A critical review of congenital phantom limb cases and a developmental theory for the basis of body image. Conscious Cogn 2006; 15: 310-22.

32. Brugger P, Kollias SK, Müri G, Hepp-Reymond M-C, Regard M. Beyond remembering: Phantom sensations of congenitally absent limbs. Proc Natl Acad Sci USA 2000; 97: 6167-72.

33. Gallagher S, Butterworth GE, Lew A, Cole J. Handmouth coordination, congenital absence of limb, and evidence for innate body images. Brain Cogn 1998; 38 : 53-65.

34. Ramachandran VS, Hirstein W. The perception of phantom limbs. Brain 1998; 121: 1603-30.

35. Gallese V, Goldman A. Mirror neurons and the simulation theory of mind-reading. Trends Cogn Sci 1998; 2: 493-501.

36. Hepper PG, McCartney GR, Shannon EA. Lateralised behaviour in first trimester human foetuses. Neuropsychologia 1998; 36: 531-4.

37. Botvinick M, Cohen J. Rubber hands feel touch that eyes see. Nature 1998; 391: 756.

38. Pavani F, Spence C, Driver J. Visual capture of touch: Out-of-the-body experiences with rubber gloves. Psychol Sci 2000; 11: 353-9. 